\title{
Increased testis growth and sperm production in adult rats following transient neonatal goitrogen treatment: optimization of the propylthiouracil dose and effects of methimazole
}

\author{
P. S. Cooke ${ }^{1}$, J. D. Kirby ${ }^{2}$ and J. Porcelli ${ }^{1}$ \\ ${ }^{1}$ Department of Veterinary Biosciences, University of Illinois, 2001 S. Lincoln Ave, Urbana, IL 61801, \\ USA; and ${ }^{2}$ Department of Urology, Northwestern University Medical School, Chicago, IL 60611,
} USA

\begin{abstract}
Treatment of male rat pups with the reversible goitrogen 6-propyl-2-thiouracil (PTU), administered by adding $0.1 \%$ PTU to the mother's drinking water from birth to day 25 , increases their testis size and daily sperm production (DSP) at 160 days of age by up to $80 \%$ and $140 \%$, respectively. The purpose of this study was to examine the effectiveness of various concentrations of PTU and determine the PTU dose that would maximize testis growth while minimizing side effects such as decreased maternal water consumption and decreased pup growth. Whether this effect was specific to PTU was determined by evaluating the effects of another commonly used goitrogen, methimazole (MMI), in increasing adult testis size and sperm production. Dams were given PTU $(0.1-0.0004 \% \mathrm{w} / \mathrm{v})$ or $0.025 \% \mathrm{MMI}$ $(\mathrm{w} / \mathrm{v})$ in their water from birth to day 25 post partum, then given no further treatment. Thyroxine concentrations were measured in all groups of pups at 25, 35 and 45 days, and testis weight and DSP were determined at 90 days of age. At 25 days of age, thyroxine concentrations were maximally decreased by PTU treatments of $0.0015 \%$ or greater; less severe decreases were produced by $0.0004 \%$ PTU or $0.025 \%$ MMI. Thyroxine concentrations increased in all treated groups at day 35 compared with day 25 , and returned to normal by day 45 . At 90 days of age, testis weight was increased by about $40 \%$ in rats whose mothers had been treated with doses of $0.006 \%$ PTU or greater, whereas testis weights in groups given 0.0015 and $0.0004 \%$ PTU or $0.025 \%$ MMI were increased 31,15 and $18 \%$, respectively. Similarly, DSP showed maximal increases at PTU doses of $0.006 \%$ or greater, whereas lower doses or $\mathrm{MMI}$ produced gradations of the maximal increase. At 90 days, body weights in the 0.1 and $0.025 \%$ PTU groups were reduced by approximately $25 \%$. Despite the maximal increase in testis size and DSP, body weights in the $0.006 \%$ PTU group showed a smaller decrease $(16 \%)$. Lower PTU doses produced smaller body weight decrements, and the body weight decrease in the MMI group (11\%) was similar to that in the $0.0004 \%$ PTU group. In summary, a $0.006 \%$ PTU concentration is optimal for maximally increasing testicular growth and DSP at 90 days while minimizing side effects; lower concentrations of PTU $(0.0015$ and $0.0004 \%)$ produce gradations of the maximal effect. Neonatal MMI administration was also effective in increasing adult testis size and DSP, although the potency of this drug is less than PTU and parallels its decreased potency compared with PTU in reducing thyroxine concentrations.
\end{abstract}

\section{Introduction}

We have previously shown that neonatal goitrogen treatment can increase adult testis size and sperm production. In this system, rats are made hypothyroid from birth until day 25 by adding the goitrogen 6-propyl-2-thiouracil (0.1\%; PTU) to the mother's drinking water. The PTU ingested by the mother is transferred through the milk to the pups, where it causes severe hypothyroidism (Blake and Henning, 1985; Tamasy et

Received 7 May 1992 al., 1986; Kirby et al., 1992). Body and organ growth of the pups are markedly suppressed by this regimen of PTU treatment (Meisami, 1984; Cooke and Meisami, 1991; Cooke, 1991). Upon cessation of PTU administration, treated rats begin to grow rapidly; their body weights eventually parallel controls, but always remain 15-25\% lower than normal (Meisami, 1984; Cooke and Meisami, 1991; Cooke et al., 1992). Testis weights and daily sperm production (DSP) are increased by 40 and $83 \%$, respectively, at 90 days of age (Cooke and Meisami, 1991; Cooke et al., 1991, 1992), and maximal increases in these parameters are obtained at 160 days of age, when testis weight 
and DSP are about 80 and $140 \%$ greater than normal, respectively (Cooke and Meisami, 1991; Cooke, 1991; Cooke et al., 1991).

The $0.1 \%$ PTU dose used in all of these experiments maximally suppresses thyroxine $\left(\mathrm{T}_{4}\right)$ in the pups and produces a smaller decrease in triiodothyronine $\left(\mathrm{T}_{3}\right)$ concentrations (Tamasy et al., 1986; Kirby et al., 1992). Concentrations of $\mathrm{T}_{4}$ begin to rise after the cessation of PTU treatment, and in pups treated from birth to day $25, T_{4}$ concentrations return to normal at about day 45-50 (Kirby et al., 1992). Conversely, thyroidstimulating hormone concentrations are increased eight-fold compared with controls in PTU-treated pups at 25 days of age, then decline rapidly and, like $\mathrm{T}_{4}$ concentrations, reach normal concentrations by about day 45-50 (Kirby et al., 1992).

Neonatal PTU treatment of rats has been used to study thyroid hormone effects on many aspects of growth, brain maturation and cognitive functions. In the studies in which hypothyroidism in the neonates was induced by adding PTU to the mother's water, a wide variety of concentrations ranging from 0.1 to $0.01 \%$ have been used (Meisami, 1984; Blake and Henning, 1985; Tamasy et al., 1986; Behnam-Rassoli et al., 1991), and the relationship between the PTU concentration given in the mother's water and the ultimate degree of hypothyroidism in the pups has been determined (Blake and Henning, 1985).

One important aspect of the PTU effect on adult testis and function that has not been previously addressed is the possible dependence of this phenomenon on the degree of hypothyroidism during the neonatal treatment period. In other words, is maximal suppression of thyroid function during the neonatal period necessary to produce this effect, or will lower doses of PTU and smaller degrees of hypothyroidism also cause increases in testicular weight? If lower doses of PTU can produce this effect, another equally important question is whether there is a threshold dose of PTU that will yield maximal effects on the testes or whether lower doses will produce gradations of the maximal PTU effect.

Neonatal PTU treatment produces a range of undesirable side effects such as decreased body weight, decreased vigour of pup suckling, delayed weaning and delayed ontogeny of intestinal digestive enzymes (Henning, 1978; Narayanan et al., 1982; Blake and Henning, 1985; Cooke and Meisami, 1991). Furthermore, our previous experience with PTU treatment administered through the mother's water suggested that this treatment may decrease maternal water consumption, which has the potential to affect pup nutrition and growth adversely. Determination of the optimal PTU dose that produces substantial or maximal testis increases while minimizing side effects would therefore be very useful.

The aim of the present study was to determine the relationship between the degree of neonatal hypothyroidism and the ultimate degree of increased testis growth and function and to optimize the protocol for obtaining this effect. We examined the effects of various doses of PTU on the degree of hypothyroidism in the pups during treatment, the rapidity of their return to euthyroidism after the treatment was stopped, the level of maternal water consumption and the ultimate induction of increased testis weight and sperm production.

Neonatal treatment with another commonly used goitrogen, methimazole (MMI) has been shown to produce pronounced decreases in testicular growth and maturation during the treatment period (Palmero et al., 1989; Francavilla et al., 1991). Our work showing that neonatal PTU treatment stimulates adult testis size and function suggested that transient neonatal MMI treatment might have similar stimulatory effects on the adult testis despite its documented inhibitory actions on early testis development. We therefore examined the ability of neonatal MMI treatment to increase adult testis weight and sperm production.

\section{Materials and Methods}

\section{Animals and treatments}

Sprague-Dawley rats derived from parental stock purchased from Harlan (Indianapolis, IN) were bred and maintained in our animal colony as described previously (Cooke and Meisami, 1991; Cooke et al., 1992). After birth, pups were sexed and weighed. Only male pups were retained, and litter size was adjusted to 6-8. Pups were weighed weekly and maintained in accordance with the NIH Guide for the Care and Use of Laboratory Animals.

Some litters were randomly designated as controls; control mothers and their pups were untreated and received food and water ad libitum. Other litters were made hypothyroid by adding various doses of PTU (Sigma, St Louis) to the mother's water beginning immediately after birth so that the final concentration ranged from 0.1 to $0.0004 \%(\mathrm{w} / \mathrm{v})$; at least two litters were used for each PTU dose tested. The $0.0004 \%$ dose was chosen as the lowest amount because previous work has shown that $\mathrm{T}_{4}$ concentrations are only slightly decreased by administration of lower concentrations such as $0.0001 \%$ (Blake and Henning, 1985). Additional litters were given MMI $(0.025 \%$; Sigma, St Louis) in the mother's water. This dose of MMI and the method of administration have been used extensively and are sufficient to produce the maximal degree of hypothyroidism that can be obtained with this drug (Morreale de Escobar, 1988; Palmero et al., 1989; Munoz et al., 1991; Francavilla et al., 1991; Nanto-Salonen et al., 1991). Both MMI and PTU are reversible goitrogens that inhibit $\mathrm{T}_{4}$ synthesis; PTU also inhibits peripheral deiodination of $T_{4}$ to $T_{3}$ (Engler et al., 1982).

For treated litters, food and the PTU-containing water were offered ad libitum. PTU has a bitter taste, so diet cherry Kool-Aid was added to the PTU-containing water to increase palatability (Cooke and Meisami, 1991). The use of different concentrations of PTU in the mother's water raised the possibility that these solutions might have different degrees of palatability, and that different levels of water intake amongst the different treatment groups could confound attempts to administer graded doses of PTU to the various litters. Daily maternal water consumption was monitored in all groups from birth to day 25 to assess whether water consumption was influenced by the addition of different PTU concentrations or MMI.

At 25 days of age, PTU or MMI treatment was discontinued and pups were weaned and housed 3-4 per cage. Pups were given tap water and food ad libitum thereafter. Blood samples were taken from all groups by cardiac puncture at 25,35 and 45 days to evaluate the severity of the hypothyroidism induced by 
the various treatments and the rapidity of the recovery in $T_{4}$ concentrations when these treatments were discontinued. The serum was separated by centrifugation at $500 \mathrm{~g}$ for $2 \mathrm{~min}$ and frozen at $-20^{\circ} \mathrm{C}$ for determination of $\mathrm{T}_{4}$. At 90 days of age, control and treated rats were decapitated, and the testes were weighed and then frozen for subsequent determination of daily sperm production (Cooke et al., 1991).

\section{Measurement of daily sperm production and thyroid hormone concentrations}

To determine daily sperm production (DSP), testes were decapsulated, weighed and then homogenized (Robb et al., 1978; Cooke et al., 1991). Step 17-19 spermatids (Stage IVVIII), which survive this homogenization, were counted using a haemocytometer to obtain total number of spermatids per testis, as previously described (Robb et al., 1978; Cooke et al., 1991). In the Sprague-Dawley rat, developing spermatids spend approximately 6.3 days in steps 17-19 during spermatogenesis (Clermont and Harvey, 1965). Thus, the values for the numbers of spermatids per testis were divided by 6.3 to obtain DSP. The efficiency of sperm production (DSP $\mathrm{g}^{-1}$ testis) was obtained by dividing DSP by the weight of the decapsulated testis.

Total $T_{4}$ and $T_{3}$ concentrations were determined as previously described (Cooke and Meisami, 1991). Sensitivity of the $\mathrm{T}_{4}$ assay was $0.6 \mu \mathrm{g} \mathrm{dl}^{-1}$, with intra-assay and interassay coefficients of variation of $3.0 \%$ and $3.5 \%$, respectively.

Sensitivity of the $\mathrm{T}_{3}$ assay was $10 \mathrm{ng} \mathrm{dl}^{-1}$, with intra-assay and interassay coefficients of variation of $3.0 \%$ and $9.0 \%$, respectively. Equal aliquots of serum from each individual in a litter were combined and the $T_{4}$ and $T_{3}$ concentrations of the pooled samples from that litter were determined by duplicate measurements; at least two litters were used for each treatment.

\section{Statistical analysis}

Data were analysed using a one-way ANOVA. Several tests of the data were performed to ensure that the data conformed to the assumptions of the one-way ANOVA. Bartlett's test was used to test for homogeneity of variances and logarithmic transformation (Winer, 1971) was used where variances were not homogeneous. The Tukey-Kramer HSD test (Stoline, 1981) was used to identify significant $(P<0.05)$ differences between means.

\section{Results}

\section{Testis weights}

Testis weights of 90-day-old rats that were untreated (control) or given MMI or various doses of PTU during the first 25 days of life are shown (Fig. 1). Testis weights were similar in groups given $0.1,0.025,0.012$ and $0.006 \%$ PTU; in all cases the increases in testis weight compared with controls were about $40 \%$. Treatment with lower PTU doses such as 0.0015 and $0.0004 \%$ produced smaller but significant $(P<0.05)$ increases in testis weight ( 31 and $15 \%$, respectively) which were

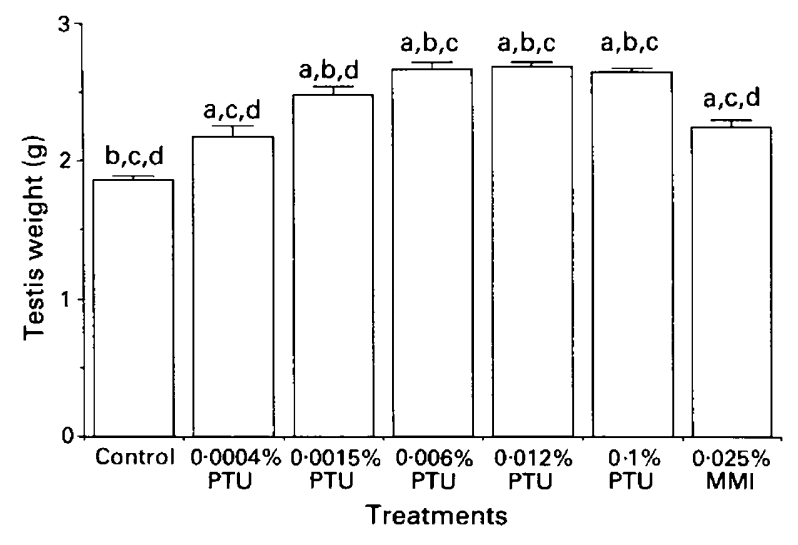

Fig. 1. Wet weight of testes from 90-day-old control rats and rats treated with various doses of propylthiouracil (PTU) or methimazole (MMI) from birth to day 25. Bars represent means \pm SEM weights of single testes ( $n$ was $\geq 20$ for all groups). Testis weights that were significantly $(P<0.05)$ different from controls are denoted by an (a) above the bar, those different from the $0.0004 \%$ PTU group by a (b), those different from the $0.0015 \%$ PTU group by a (c) and those different from the $0.1 \%$ PTU group by a (d).

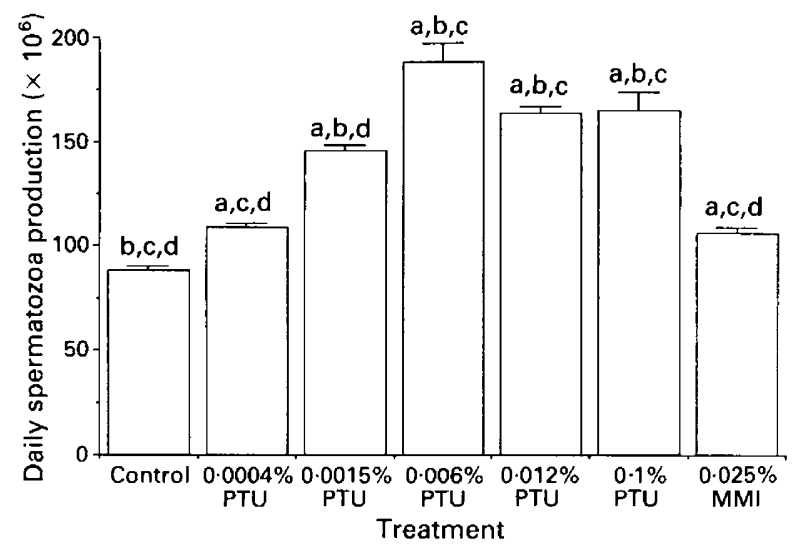

Fig. 2. Daily sperm production (DSP) at 90 days of age in control rats and rats treated with various doses of propylthiouracil (PTU) or methimazole (MMI) from birth to day 25. Data are presented as means \pm SEM of duplicate determinations from individual testes ( $n$ was $\geq 16$ for all groups except the $0.012 \%$ PTU dose, where $n=8$ ). DSP values that were significantly $(P<0.05)$ different from controls are denoted by an (a) above the bar, those different from the $0.0004 \%$ PTU group by a (b), those different from the $0.0015 \%$ PTU group by a (c) and those different from the $0.1 \%$ PTU group by a (d).

proportional to the dose of PTU administered. MMI (0.025\%) produced a significant increase in testis weight $(18 \%)$ similar in magnitude to the $0.0004 \%$ PTU dose.

\section{Sperm production}

PTU doses of $0.006 \%$ or greater produced increases in DSP at 90 days that were equal to or greater than those normally obtained with the 0.1\% PTU dose (Fig. 2). The lower doses of 


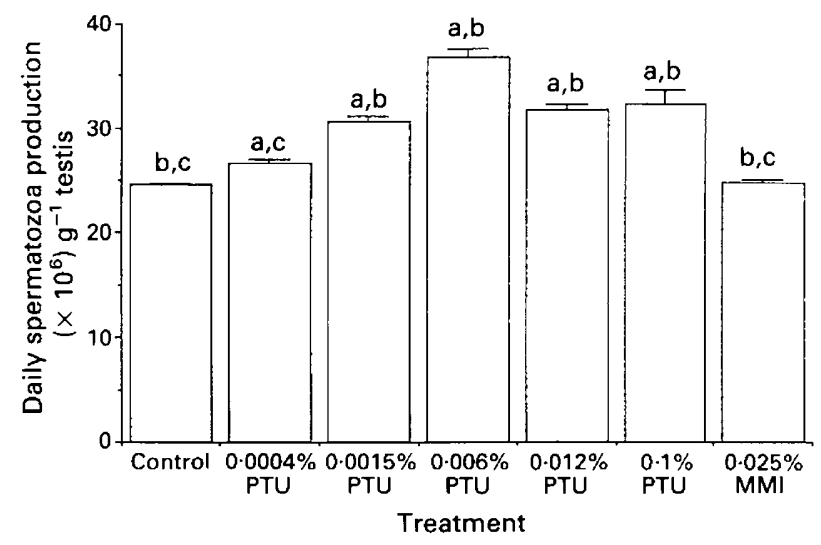

Fig. 3. Efficiency of sperm production (daily sperm production per $g$ testis) at 90 days of age in control rats and rats treated with various doses of propylthiouracil (PTU) or methimazole (MMI) from birth to day 25. Data are presented as means \pm SEM of duplicate determinations from individual testes ( $n$ was $\geq 16$ for all groups except the $0.012 \%$ PTU dose, where $n=8$ ). Efficiency of sperm production values that were significantly $(P<0.05)$ different from controls are denoted by an (a) above the bar, those different from the $0.0004 \%$ PTU group by a (b) and those different from the $0.1 \%$ PTU group by a (c).

PTU (0.0015 and $0.0004 \%)$ produced gradations of the DSP increase seen with the higher doses; MMI produced an increase in DSP which was similar to that seen with the lowest dose $(0.0004 \%)$ of PTU.

The efficiency of sperm production (DSP $\mathrm{g}^{-1}$ testis) was increased by $25-35 \%$ in animals treated with a PTU dose of $0.006 \%$ or greater (Fig. 3). The $0.0015 \%$ PTU dose increased DSP $\mathrm{g}^{-1}$ testis by $8 \%(P \leq 0.05 \%)$ compared with controls. Efficiency of sperm production was not different from that observed in controls in rats given $0.0004 \%$ PTU or $0.025 \%$ MMI.

\section{Thyroid hormone concentrations}

At 25 days of age, concentrations of $T_{4}$ were markedly suppressed by PTU in all treatment groups (Fig. 4). PTU treatment at a dose of $0.0015 \%$ or greater suppressed $\mathrm{T}_{4}$ concentrations to below the limit of detection of the assay $\left(0.6 \mu \mathrm{g} \mathrm{dl}^{-1}\right)$ at this age, whereas the $0.0004 \%$ dose produced a less severe suppression of $\mathrm{T}_{4}$ concentrations. The suppression of $\mathrm{T}_{4}$ concentrations by the $0.025 \% \mathrm{MMI}$ dose was less severe than that produced by an equivalent or even several times lower PTU dose, and was similar to that observed with $0.0004 \%$ PTU. $T_{4}$ concentrations increased markedly between the cessation of PTU or MMI treatment (day 25) and day 35 , with $\mathrm{T}_{4}$ concentrations in groups treated with MMI or the lower doses of PTU returning to near-normal, while the suppression of $T_{4}$ in groups given the higher doses of PTU persisted to a greater degree. $T_{4}$ concentrations in all groups had recovered to normal or near-normal by day 45 .

In contrast to the almost total suppression of $\mathrm{T}_{4}$ by PTU, $\mathrm{T}_{3}$ concentrations were reduced by only $60-70 \%$ at 25 days in rats treated with PTU doses of $0.006 \%$ or greater, whereas MMI and the $0.0004 \%$ PTU produced significant decreases of slightly less than $50 \%$ in $\mathrm{T}_{3}$ concentrations (data not shown).

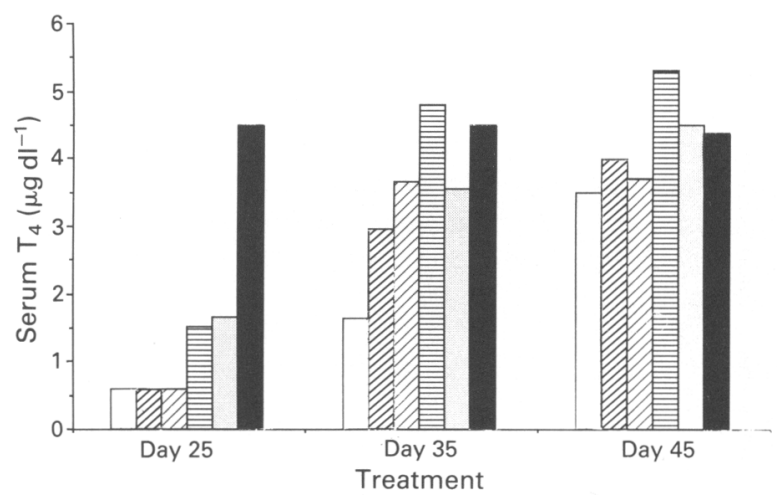

Fig. 4. Thyroxine $\left(T_{4}\right)$ concentrations in serum from $(\boldsymbol{\square})$ control rats and rats treated with various doses of propylthiouracil (PTU) or methimazole (MMI): ( $\square$ ) $0.1 \%$ PTU, ( $\$ ) $0.012 \%$ PTU, (も) $0.006 \%$ PTU, (目) $0.0004 \%$ PTU and (國) $0.025 \%$ MMI. Each bar represents the mean of two serum pools, each of which consisted of equal amounts of serum from all of the animals in one litter. At 25 days of age, PTU treatments of $0.0015 \%$ PTU or greater produced maximal suppression of serum $\mathrm{T}_{4^{\prime}}$, while lower PTU doses $(0.0004 \%)$ or $0.025 \%$ MMI produced lesser degrees of hypothyroidism. $\mathrm{T}_{4}$ concentrations were increased by 35 days of age compared with 25 days in all groups and $\mathrm{T}_{4}$ concentrations had returned to normal or near-normal in all groups by 45 days of age.

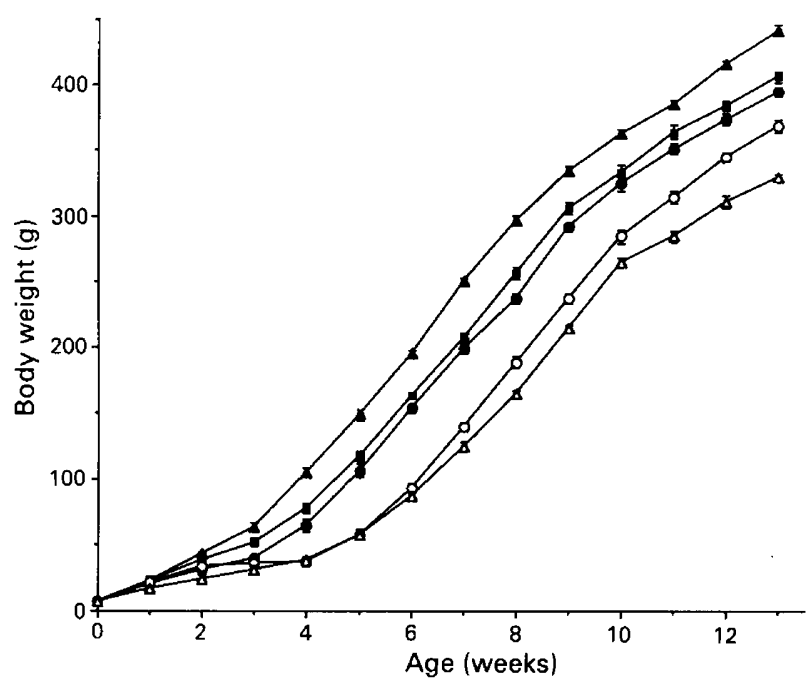

Fig. 5. Growth of rats that were $(\boldsymbol{\Delta})$ untreated (control) compared with those given various doses of propylthiouracil (PTU) or methimazole (MMI) from birth to day 25 ( ) $0.0004 \% \mathrm{PTU},(\bigcirc) 0.025 \% \mathrm{MMI},(\mathrm{O})$ $0.006 \%$ PTU and $(\triangle) 0.1 \%$ PTU. Data points are means \pm SEM. For all groups, at least two litters were used for all points. At 90 days of age, the body weights of rats given the $0.0004 \%$ PTU or the $0.025 \% \mathrm{MMI}$ were not different from each other. All other groups were significantly $(P<0.05)$ lower than controls and were different from each other.

\section{Growth of control and treated rats}

Increasing concentrations of PTU in the mother's water produced concomitant decreases in pup growth during the treatment period, reaching maximal levels at PTU doses of 0.025 and $0.1 \%$ (Fig. 5). MMI (0.025\%) also decreased body 


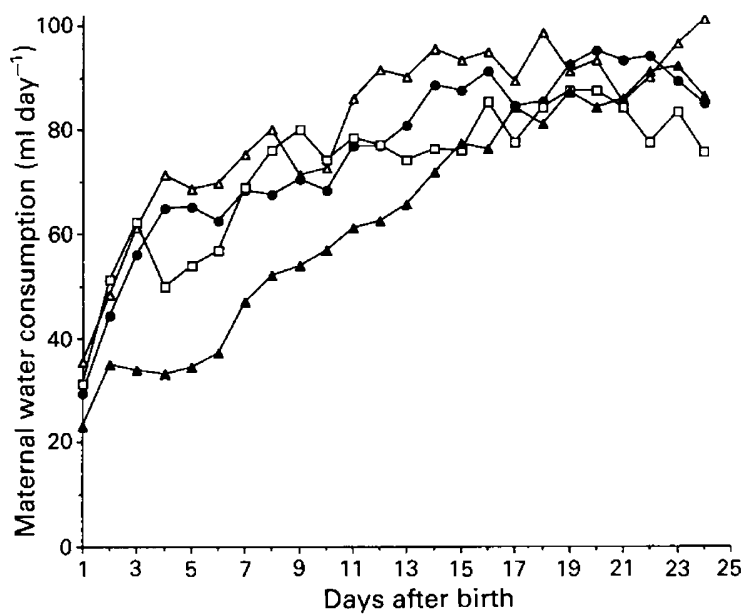

Fig. 6. Daily maternal water consumption during the suckling period in $(\triangle)$ control rats and rats treated with various doses of propylthiouracil (PTU) or methimazole (MMI) (O) 0.0004\% PTU, ( $\square$ ) $0.006 \%$ PTU and $(\boldsymbol{\Delta}) 0.1 \%$ PTU. Water consumption in the $0.025 \%$ PTU group (not shown) was similar to that of the $0.1 \%$ PTU group, but when the PTU dose was decreased to $0.012 \%$ (not shown) or $0.006 \%$, water consumption was greatly increased. Water consumption in the groups given $0.004 \%$ PTU or $0.025 \%$ MMI (not shown) was only minimally suppressed. Values represent means of at least two litters for all data points.

growth, but the severity of the weight decreases was markedly less than that seen with the higher doses of PTU. Rats treated with either MMI or various doses of PTU began growing rapidly 5-7 days after PTU withdrawal, initially exceeding then subsequently paralleling the growth rate of the controls. However, body weights of all PTU-treated groups remained significantly less than controls at all ages up to 90 days. The body weight differences between the different treatment groups also persisted (Fig. 5). At 90 days of age, body weight decrements in the $0.1,0.025$ (not shown), 0.012 and $0.006 \%$ PTU groups were $24,23,17$ and $16 \%$, respectively. Body weights in pups given lower doses of PTU, 0.0015 and $0.0004 \%$, were decreased by 20 and $8 \%$, respectively, at 90 days. The body weight decreases in MMI-treated rats also persisted throughout the experiment; the decrease at day $90(11 \%)$ was similar to that seen with the $0.0004 \%$ PTU dose.

\section{Maternal water consumption during nursing}

Water consumption by control mothers increased rapidly during the early suckling period, plateauing at about $100 \mathrm{ml}$ day $^{-1}$ at 2 weeks post partum (Fig. 6). Water consumption by mothers offered water containing 0.1 or $0.025 \%$ PTU was markedly reduced immediately after birth and remained less than that of control mothers until almost the end of the suckling period. This decrease was most apparent during the early postnatal period. For example, water consumption in control mothers was $106 \%$ greater than that of mothers given $0.1 \%$ PTU 5 days after birth. Decreasing the PTU content of the mother's water to 0.012 or $0.006 \%$ increased maternal water consumption, especially during the early neonatal period, when mothers given these doses drank $35-70 \%$ more water per day than the mothers given $0.1 \%$ PTU, despite the fact that pup weights in these various treatment groups were not substantially different during this period.

The large decrease in water consumption seen during the early suckling period in the mothers treated with 0.1 or $0.025 \%$ PTU became less pronounced with advancing age (Fig. 6), ultimately reaching a similar plateau as the controls, but at a later age. Water consumption in MMI-treated mothers (data not shown) was reduced only slightly compared with controls during the suckling period, and the pattern of water consumption in the MMI-treated mothers closely paralleled the small decrement seen in mothers given the $0.0004 \%$ PTU dose.

\section{Discussion}

The results of the present study show that low concentrations of PTU produce a dose-dependent increase in testis size and sperm production at 90 days until a maximal plateau is reached, at PTU doses of $0.006 \%$ or greater. It was not previously known whether doses lower than the $0.1 \%$ concentration previously used would produce gradations of the maximal testicular effect or whether there was some threshold PTU dose that would produce the full increase in testicular size and function whereas lower doses would be totally ineffective; the present results clearly show that the former is correct. The ability of lower PTU doses $(0.0015$ and $0.0004 \%)$ to produce gradations of the maximal testis increase provides a unique method whereby a desired degree of increased adult testicular weight and DSP can be obtained simply by varying the neonatal PTU dose administered, and will allow a degree of experimental control of these parameters that has not been possible previously.

A neonatal PTU dose as low as $0.006 \%$ is the minimum one that can maximally increase adult testis growth and sperm production at 90 days. Thus, the PTU concentration previously administered to nursing mothers to increase testis size and function in the male offspring, $0.1 \%$, can be reduced more than 16 -fold to $0.006 \%$ without diminishing the increase in testis size and function seen in adult male offspring. Like the $0.1 \%$ dose, the lower dose $(0.006 \%)$ maximally suppresses $T_{4}$ concentrations during treatment. However, $\mathrm{T}_{4}$ concentrations in rats treated with the $0.006 \%$ dose recover faster after cessation of PTU treatment than do those of the pups whose mothers were treated with the $0.1 \%$ dose. This more rapid return to euthyroidism with the $0.006 \%$ PTU dose suggests that testes of these animals may be more maturationally advanced at 90 days than those of the $0.1 \%$ group; therefore, despite maximal testis increases in the $0.006 \%$ PTU group at 90 days it is not clear from our data whether ultimate testis size attained in this group at final maturity will equal that seen with higher doses such as $0.1 \%$.

In contrast to the similar increases in testicular weight and function seen in male rats treated with doses of $0.006 \%$ or greater, the overall body weight decreases were significantly lessened in offspring of mothers given the $0.006 \%$ PTU dose compared with those given the $0.1 \%$ dose. Similarly, maternal water consumption, which is markedly reduced in mothers given the $0.1 \%$ PTU dose, was significantly improved in mothers given the $0.006 \%$ PTU dose, especially during the 
neonatal period. The ability of the $0.006 \%$ dose to maximize increase in testis size and sperm production while allowing a faster return to euthyroidism following PTU treatment and minimizing side effects such as decreased water consumption and body weight gain indicates that the $0.006 \%$ dose is optimal for producing this effect.

The mechanism by which the decreased PTU doses (for example $0.006 \%$ ) lead to less severe body weight decreases in the male offspring of the treated mothers than higher doses such as $0.1 \%$ is not clear. One obvious explanation would be that the higher PTU doses produce more severe thyroid hormone suppression and concomitant decreases in body weight gain than does the $0.006 \%$ dose. Since PTU doses of $0.006 \%$ or greater suppress $\mathrm{T}_{4}$ to below the sensitivity of the assay used in the present study $\left(0.6 \mu \mathrm{g} \mathrm{dl}^{-1}\right)$, this question cannot be answered from our data. The more rapid return to euthyroidism seen in the pups given the $0.006 \%$ PTU dose compared with those given the $0.1 \%$ dose is almost certainly a contributory factor to the greater growth of these pups after PTU treatment is stopped at 25 days.

The increased growth in pups treated with the $0.006 \%$ dose compared with the $0.1 \%$ dose probably also reflects pup nutrition. The results on water intake by dams given PTU represent the first demonstration that this treatment can substantially decrease maternal water consumption at higher PTU concentrations, including $0.1 \%$, the dose previously used in all studies on the ability of PTU to increase adult testis size (Cooke and Meisami, 1991; Cooke et al., 1992). The large decrease in maternal water consumption during the first 2 weeks of nursing by mothers given water containing $0.1 \%$ PTU compared with that of mothers given tap water must logically reduce the amount of milk available and consequently the amount of nutrition available to the pups. Nursing mothers are reticent to drink PTU-containing water owing to its bitter taste even when an agent is used to mask it. The milk of these mothers, which contains significant amounts of PTU, may also be somewhat unpalatable to the developing young and this may decrease the amount of pup nursing. The lower doses of PTU (such as $0.006 \%$ ) may then lead to both increased milk production secondary to greater maternal water consumption and increased pup nursing owing to the lower concentration of PTU in the milk, eventually resulting in improved pup growth.

The substantial differences in water consumption among the dams given different PTU doses indicate that total PTU intake in the different groups does not directly correlate with the concentration of PTU placed in the dam's water. For example, water consumption by dams given $0.012 \%$ PTU is more than $60 \%$ greater than that in dams given $0.025 \%$ PTU over the first week of life. Owing to increased water consumption, total PTU intake by the dams in the group given the lower PTU concentration is therefore only about $20 \%$ less than in the group given water that contained a two-fold higher PTU concentration. Maternal water consumption has not been considered in previous studies of the different effects on the pups of various PTU doses administered through the mother's water (Blake and Henning, 1985), but the present results underscore the desirability of taking this parameter into account in these types of study to obtain more accurate information on the total dose of PTU that is being ingested by dams presented with water containing different PTU doses.
Extensive previous results have shown that neonatal MMI treatment markedly inhibits development of Sertoli and germ cells and the testis as a whole. For example, neonatal MMI administration significantly decreases testis weight, seminiferous tubule diameter and the number of germ cells per tubule (Francavilla et al., 1991). Likewise, Sertoli cell development is retarded as evidenced by the delayed appearance of the tubular lumen and of cytoplasmic lipids in the Sertoli cells (Francavilla et al., 1991). Furthermore, the normal postnatal increase in Sertoli cell production of androgen-binding protein and lactate is inhibited (Palmero et al., 1989), and the absence of thyroid hormones reduces production of IGF- 1 by neonatal Sertoli cells (Palmero et al., 1990).

Despite the documented litany of inhibitory effects of early hypothyroidism on testicular development, the present data clearly show that transient neonatal MMI treatment is actually stimulatory to adult testicular size and function. Not only are the developmental deficits caused by this treatment reversible, but also MMI, like PTU, must induce testicular or systemic (possibly endocrine) changes that result in permanently increased testis size and function if the animal is allowed to return to euthyroidism and develop normally. However, both FSH and LH are reduced during PTU treatment and stay low throughout subsequent life (Kirby et al., 1992) and testosterone concentrations are normal in adult rats that were treated neonatally with PTU (Cooke and Meisami, 1991; Kirby et al., 1992). The increased testis growth and function in adult animals following neonatal goitrogen treatment is therefore not due to increased concentrations of circulating gonadotrophin or testosterone, but apparently involves increased Sertoli cell proliferation (Cooke and Hess, 1992; Hess and Cooke, 1992) which may result from the lack of the normal maturational effects of thyroid hormones on these cells during neonatal development (Jannini et al., 1990; Palmero et al., 1992).

Although neonatal MMI treatment increases adult testis size and function, PTU is much more potent than MMI in this respect. The greater effect of PTU on testicular size is paralleled by the greater potency of PTU for inducing hypothyroidism in rats, as shown both by our present work and earlier literature (Palmero et al., 1989; Nanto-Salonen et al., 1991; Kirby et al., 1992). This observation, along with the data showing that $0.025 \% \mathrm{MMI}$ and $0.0004 \%$ PTU produce similar degrees of $T_{4}$ suppression and almost identical increases in testis size and function during adulthood, suggests that the degree of hypothyroidism in the developing rat is directly but inversely correlated with the magnitude of the adult increase in testis size and function.

The authors would like to thank N. Arambepole for her assistance in measuring sperm production. The authors thank F. Turek (Northwestern University) for his support during the completion of this research. This work was supported by NIH grant HD29376 to P. Cooke, and a USDA grant to J. Kirby.

\section{References}

Behnam-Rassoli M, Herbert LC, Howard V, Pharoah POD and Stanisstreet M (1991) Effect of propylthiouracil treatment during prenatal and early postnatal development on the neocortex of rat pups Neuroendocrinology $\mathbf{5 3}$ $321-327$ 
Blake HH and Henning SJ (1985) Effect of propylthiouracil dose on serum thyroxine, growth and weaning in young rats American Joumal of Physiology 248 R524-530

Clermont Y and Harvey SC (1965) Duration of the cycle of the seminiferous epithelium of normal, hypophysectomized and hypophysectomized-hormone treated albino rats Endocrinology 76 80-89

Cooke PS (1991) Thyroid hormones and testis development: a model system for increasing testis growth and sperm production. In The Male Germ Cell: Spermatogonium to Fertilization pp 122-132 Ed. B. Robaire. Annals of the New York Academy of Science Vol. 637

Cooke PS and Hess RA (1992) The pattern of Sertoli cell proliferation is altered in the neonatal hypothyroidism model for increased testis size Journal of Andrology 13 (Supplement) 47

Cooke PS and Meisami E (1991) Early hypothyroidism in rat increases adult testis and reproductive organ size but does not change testosterone levels Endocrinology 129 237-243

Cooke PS, Hess RA, Porcelli J and Meisami E (1991) Increased sperm production in adult rats following transient neonatal hypothyroidism Endocrinology 129 244-248

Cooke PS, Porcelli J and Hess RA (1992) Induction of increased testis growth and sperm production in the adult rat by neonatal administration of the goitrogen propylthiouracil (PTU): the critical period Biology of Reproduction 46 146-152

Engler H, Taurog A and Dorris ML (1982) Preferential inhibition of thyroxine and $3,5,3^{\prime}$-triodothyronine formation by propylthiouracil and methylmercaptoimadazole in thyroid peroxidase-catalyzed iodination of thyroglobulin Endocrinology 110 190-197

Francavilla S, Cordeschi G, Properzi G, Di Cicco L, Jannini EA, Palmero S, Fugassa E, Loras B and D'Armiento M (1991) Effect of thyroid hormone on the pre- and post-natal development of rat testis Journal of Endocrinology 129 $35-42$

Henning SJ (1978) Permissive role of thyroxine in the ontogeny of jejunal sucrase Endocrinology 102 9-15

Hess RA and Cooke PS (1992) Total number of Sertoli cells is doubled in the neonatal hypothyroidism model for increased testis size Journal of Andrology 13 (Supplement) 47

Jannini EM, Olivieri M, Francavilla S, Gulino A, Ziparo E and D'Armiento M (1990) Ontogenesis of the nuclear 3,5, $3^{\prime}$-triiodothyronine receptor in the rat testis Endocrinology $1262521-2526$

Kirby JD, Jetton AE, Cooke PS, Hess RA, Bunick D, Ackland J, Turek FW and Schwartz NB (1992) Developmental hormonal profiles accompanying the neonatal hypothyroidism induced increases in adult testis size and sperm production in the rat Endocrinology 131 559-565
Meisami E (1984) Complete recovery of growth deficits after reversal of PTUinduced postnatal hypothyroidism in the female rat: a model for catch-up growth Life Sciences 34 1487-1496

Morreale de Escobar G, Obregon MJ, Ruiz de Ona C and Escobar del Rey F (1988) Transfer of thyroxine from the mother to the rat fetus near term: effects on brain 3,5, $3^{\prime}$-triiodothyronine deficiency Endocrinology $\mathbf{1 2 2}$ 1521-1531

Munoz A, Rodriguez-Pena A, Perez-Castillo A, Ferreiro B, Sutcliffe JG and Bernal J (1991) Effects of neonatal hypothyroidism on rat brain gene expression Molecular Endocrinology 5 273-280

Nanto-Salonen K, Glasscock GF and Rosenfeld RG (1991) The effects of thyroid hormone on insulin-like growth factor (IGF) and IGF-binding protein (IGFBP) expression in the neonatal rat: prolonged high expression of IGFBP-2 in methimazole-induced congenital hypothyroidism Endocrinology 129 2563-2570

Narayanan CH, Narayanan Y and Browne RC (1982) Effects of induced thyroid deficiency on the development of suckling behavior in rats Physiology and Behavior 29 361-370

Palmero S, de Marchis M, Gallo G and Fugassa E (1989) Thyroid hormone affects the development of Sertoli cell function in the rat Joumal of Endocrinology 123 105-111

Palmero S, Prati M, Barreca A, Minuto F, Giordano G and Fugassa E (1990) Thyroid hormone stimulates the production of insulin-like growth factor 1 (IGF-1) by immature rat Sertoli cells Molecular and Cellular Endocrinology 68 6I-65

Palmero S, Prati M, de Marco P, Trucchi P and Fugassa E (1992) Development regulation of Sertoli cell metabolism by thyroid hormones Proceedings of the 7th European Workshop on Molecular and Cellular Endocrinology of the Testis Castle Elmau, Germany (Abstract)

Robb GW, Amann RP and Killian GJ (1978) Daily sperm production and epididymal sperm reserves of pubertal and adult rats Joumal of Reproduction and Fertility 54 103-107

Stoline MR (1981) The status of multiple comparisons: simultaneous estimation of all pairwise comparisons in one-way ANOVA designs American Statistician 35 134-141

Tamasy V, Meisami E, Vallerga A and Timiras PS (1986) Rehabilitation from neonatal hypothyroidism: spontaneous motor activity, exploratory behavior avoidance learning and response of pituitary-thyroid axis to stress in male rats Psychoneuroendocrinology 11 91-103

Winer BJ (1971) Statistical Principles in Experimental Design (2nd Edn), McGraw Hill, New York 